Pacific Journal of Mathematics

CONTINUOUS MEASURES, BAIRE CATEGORY, AND
UNIFORM CONTINUTY IN TOPOLOGICAL GROUPS 


\title{
CONTINUOUS MEASURES, BAIRE CATEGORY, AND UNIFORM CONTINUITY IN TOPOLOGICAL GROUPS
}

\author{
Gerald L. ITZKowitz
}

\begin{abstract}
In this paper we use an observation of $M$. Rajagopalan to show that each nondiscrete locally compact topological group can be written as a disjoint union of continuumly many closed nowhere dense $G_{\delta}$ sets. This observation also enables us to give a new constructive proof of a theorem of Kister. We show that in a nondiscrete noncompact locally compact group it is always possible to construct a bounded continuous function that is not left uniformly continuous. Finally this construction motivates a similar construction which yields examples of functions in $\operatorname{LUC}(G)$ but not in $\operatorname{RUC}(G)$ when $G$ is a nondiscrete and nonunimodular locally compact topological group or when $G$ is a nondiscrete locally compact metric group with inequivalent right and left uniform structures.
\end{abstract}

1. Introduction. In this paper we show that a simple observation concerning continuity of the Haar measure in a locally compact topological group may be used to derive some interesting set theoretic and topological properties of such groups. This observation has been used by M. Rajagopalan in [6] to show that extremally disconnected compact topological groups are discrete. Throughout this paper we shall assume that the continuum hypothesis holds.

Our underlying simple observation is the following one.

1.1 Observation. If $G$ is a locally compact nondiscrete topological group, and if $\lambda$ is left Haar measure then

(i) $\lambda\{x\}=0$ for each $x \in G$,

(ii) there exists a nested sequence $\left\{U_{n}: n=1,2, \ldots\right\}$ of neighborhoods of the identity $e \in G$ such that $\lambda\left(U_{n}\right) \rightarrow 0$.

In the sequel we will be employing the terms nowhere dense, first category, and second category, with their usual meaning.

Definition. $\quad M$ is of the second category at $x \in M$ if $M \cap U$ is of the second category for each neighborhood $U$ of $x$.

Obviously $M$ is of the second category in every neighborhood in $X$ if and only if $M$ is of the second category at each $x \in X$. 
2. Decomposition into nowhere dense $\boldsymbol{G}_{\delta}$ sets. The author wishes to thank the referee for pointing out the following stronger form of a theorem of Kakutani and Kodaira [5] (see also 8.7, [4]) which is proved in Halmos $64 \mathrm{G}$, [3]. His observation has as corollaries a strengthening of our original Theorem 2.2 from compactly generated groups to $\sigma$-compact groups, and the resulting more complete statement in Theorem 2.4 for all nondiscrete locally compact topological groups $G$. For completeness we give below a direct proof of the Kakutani-Kodaira Theorem suggested by the referee.

2.1. TheOREM (Kakutani-Kodaira). Let $G$ be a $\sigma$-compact, locally compact group with identity $e$. Then for every countable family $\left\{U_{n}: n=1,2\right.$, $\ldots$,$\} of neighborhoods of e, there is a compact normal subgroup N \subset G$ such that $N \subset \cap_{n=1}^{\infty} U_{n}$, and $G / N$ is metrizable and has a countable base for its open sets.

Proof. Write $G$ as an increasing union $\cup_{n=1}^{\infty} F_{n}$ of compact sets $F_{n}$. Select a sequence $\left\{V_{n}: n=1,2, \ldots\right\}$ of neighborhoods of $e$ so that $\bar{V}_{1}$ is compact, $V_{n}^{2} \subset V_{n-1} \cap U_{n}$, and such that $x V_{n} x^{-1} \subset V_{n-1}$ for all $x \in F_{n}$. Now 5.6(iv) in [2] holds and so $N=\cap V_{n}$ is a closed normal subgroup and hence also compact. It now follows exactly as in 8.7[4] that $\left\{\phi\left(V_{n}\right): n=1\right.$, $2, \ldots\}$ is a countable base at $N$ in $G / N$, where $\phi$ is the natural map of $G$ onto $G / N$. Thus by 8.3 [4], $G / N$ is metrizable. Also since $G=\cup_{n=1}^{\infty} F_{n}$ and $\phi$ is continuous, $G / N$ must be $\sigma$-compact and hence Lindelö. Thus $G / N$ has a countable base for its open sets.

2.2 THEOREM. Every infinite o-compact locally compact nondiscrete topological group $G$ may be written as a disjoint union of continuumly many nowhere dense compact $G_{\delta}$ sets.

Proof. Choose a nested sequence $\left\{U_{n}: n=1,2, \ldots\right\}$ of open neighborhoods of $e \in G$, such that $\lambda\left(U_{n}\right) \rightarrow 0$. By Theorem 2.1 there is a compact normal subgroup $N \subset G$ such that $N \subset \cap U_{n}$, and $G / N$ is metrizable and has a countable base for its open sets. Therefore $\operatorname{card}(G / N) \leq \mathrm{c}$. However $G / N$ is not discrete since $N$ is not open $(\lambda(N)=0)$. But then card $(G / N) \geq$ c. (See Hewitt and Ross[4], 4.26, for an interesting proof of this fact.). Clearly each $\tilde{x} \in G / N$ is a compact $G_{\delta}$. Hence so is each coset of $N$ in $G$. Finally since

$$
G=U\left\{x N: x \in \varphi^{-1}(\tilde{x}), \tilde{x} \in G / N, \varphi \text { the quotient map }\right\},
$$

the theorem follows .

Our method of proof allows us to deduce the following. 
2.3. THEOREM. Let $G$ be a locally compact topological group. Then the following are equivalent:

(i) $G$ is not discrete

(ii) $G$ contains a nowhere dense compact $G_{\delta}$ subgroup $N$.

(iii) There exists a nested sequence $\left\{U_{n}\right\}$ of neighborhoods of $e \in G$ such that $\lambda\left(U_{n}\right) \rightarrow 0$, where $\lambda$ is Haar measure.

Proof. (i) $\Rightarrow$ (iii) is our observation 1.1, and (ii) $\Rightarrow$ (i) since $e \in N$ is nowhere dense and hence not open. Thus we need only show that (ii) follows from (iii).

Choose a sequence of neighborhoods $\left\{V_{n}: n=1,2, \ldots\right\}$ of $e$ such that $V_{n}^{2} \subset V_{n-1} \cap U_{n}$. Then since $\bar{V}_{n} \subset V^{2}$ it follows that

$$
N=\prod_{n=2}^{\infty} V_{n} \subset \prod_{n=2}^{\infty} \bar{V}_{n} \subset \prod_{n=2}^{\infty} V_{n}^{2} \subset \prod_{n=2}^{\infty} V_{n-1}=N \text {, }
$$

so that $N$ is closed. Clearly $N \subset \cap U_{n}$ so that $\lambda(N)=0$. Thus $N$ is nowhere dense. By Theorem 5.6 in [4] $N$ is a subgroup. Since we may assume that $\bar{U}_{1}$ is compact, our theorem is true.

2.4. THEOREM. If $G$ is a nondiscrete locally compact topological group then $G$ may be written as a disjoint union of continuumly many closed nowhere dense $G_{\delta}$ sets.

Proof. $G$ contains an open nondiscrete $\sigma$-compact subgroup $J$ to which Theorem 2.2 applies. Thus $J$ may be written as a disjoint union $\cup_{\alpha \in A} F_{\alpha}$ where card $(A)=\mathfrak{c}$ and each $F_{\alpha}$ is a compact nowhere dense $G_{\delta}$ set. Let $B$ be a subset of $G$ such that $B J=G$ and such that the family $\{b J: b \in$ $B\}$ is disjoint. For each $\alpha \in A$, let $E_{\alpha}=\cup_{b \in B} b F_{\alpha}$. Then each $E_{\alpha}$ is a closed nowhere dense $G_{\delta}$ set in $G$ and $\cup_{\alpha \in A} E_{\alpha}=G$.

That Theorem 2.4 cannot be extended to read " $G$ may be written as a disjoint union of continuumly many compact nowhere dense $G_{\delta}$ sets" is evident from the following example.

EXAMPLE. Let $G=R \times G_{\circ}$ where $G_{\circ}$ is discrete, card $\left(G_{\circ}\right)>\mathfrak{c}$, and $R$ is the real numbers with the usual topology. Clearly any compact subset of $G$ is contained in a union of only finitely many of the disjoint open sets $\{R$ $\left.\times\{x\}: x \in G_{\circ}\right\} \subset G$. Thus any c compact subsets of $G$ are contained in a union of the form $E=\cup_{x \in A} R \times\{x\}$, where card $(A) \leq \mathfrak{c}$. Thus $E$ is a proper subset of $G$ and hence so is a union of any c compact subsets of $G$. 
3. Decompositions into continuumly many disjoint sets of second category. S. Ulam in 1933 [10] (and [9]), proved the following assuming the continuum hypothesis holds.

3.1. Theorem. Let $X$ be a perfect space and let $Z \subset X$ be of the second category in $X$ where card $(X)=c$. Then there exists a collection of $\mathrm{c}$ mutually disjoint sets in $Z$ which are all of the second category.

Definition. A perfect space is a complete metric space with no isolated points.

For topological groups the above theorem takes the following form.

3.2. THEOREM. Each nondiscrete $\sigma$-compact locally compact metric group $G$ may be written as a disjoint union of $\mathrm{c}$ sets each of the second category in $G$.

W. Sierpinski in [8] was able to use Ulam's theorem to prove a considerably strengthened version for the real line which we state below.

3.3. THEOREM. Each subset of the line of the second category in each interval of the line may be written as a disjoint union of $\mathrm{c}$ sets each of the second category in each interval.

We will show that the method of proof employed by Sierpinski is valid in the more general setting of locally compact, $\sigma$-compact metric spaces with no isolated points.

3.4. LEMMA. Let $X$ be a locally compact $\sigma$-compact metric space with no isolated points. If $M \subset X$ is of the second category, then there exists a neighborhood $U \subset X$ such that $M$ is of the second category at each point of $U$.

Proof. Since $X$ is a $\sigma$-compact metric space it has a countable dense set $\left\{x_{i}\right\}$ and the open balls $B_{r, i}=\left\{y: d\left(y, x_{i}\right)<r, r\right.$ rational $\}$ form a base for the topology. If the Lemma is false, then for each $B_{r, i}$ there is some $B_{r, j}$ $\subset B_{r, i}$ such that $B_{r^{\prime}, j} \cap M$ is of the first category. Let $\left\{B_{i}\right\}$ be the collection of all $B_{r, i}$ such that $B_{r, i} \cap M$ is of the first category. Then

$$
D=\complement\left(\bigcup_{i=1}^{\infty} B_{i}\right)
$$


is a closed set which contains no open set (since each open set contains a $B_{i}$ ). Thus $D$ is of the first category. But then $D \cap M$ is of the first category so that

$$
M=(D \cap M) \cup\left[\bigcup_{i=1}^{\infty}\left(M \cap B_{i}\right)\right]
$$

is a set of the first category, a contradiction.

Convention. Let $\left\{B_{i}\right\}$ denote the basis of open balls $\left\{B_{r, i}\right\}$ of rational radii for $X$.

3.5. LeMMA. Let $X$ be a locally compact $\sigma$-compact metric space with no isolated points. Let $M \subset X$ be of the second category in each open subset of $X$ and let $U \subset X$ be open. Then there exists $Q \subset \bar{U} \cap M$ of the second category such that $M-Q$ is of the second category in each neighborhood of $X$.

Proof. By the theorem of $S$. Ulam the set $\bar{U} \cap M$ contains c mutually disjoint sets each of the second category. Let $\Phi$ denote this family of sets and let $A \in \Phi$. Let $\left\{C_{i}\right\} \subset\left\{B_{i}\right\}$ be the relative basis for the open sets in $U$. Since $A$ is of the second category, there is $C_{k}$ such that $A \cap C_{k}$ is of the second category at each point in $C_{k}$.

Observe now that the family $\left\{C_{i}\right\}$ is countable though $\Phi$ is uncountable. Thus there is a closed ball $\bar{C}_{l}$ such that uncountably many members of $\Phi$ are of the second category at each point of $\bar{C}_{l}$. Let $A_{1}, A_{2} \in \Phi$ be two such sets.

Let $Q=C_{l} \cap A_{2}$. Clearly the set $Q \subset \bar{U}$ is of the second category in $\bar{U}$. Also

$$
\begin{aligned}
M-Q & =\left(M-C_{l}\right) \cup\left(M \cap C_{l}-Q\right) \\
& =\left(M-C_{l}\right) \cup\left(C_{l} \cap\left(M-A_{2}\right)\right) \supset\left(M-C_{l}\right) \cup\left(C_{l} \cap A_{1}\right)
\end{aligned}
$$

since $A_{1} \cap A_{2}=\varnothing, A_{1} \subset M, \mathrm{~A}_{2} \subset M$. Thus $M-Q$ is of the second category at each point of $C_{l}$ and at each point of $X-C_{l}$. This proves the lemma.

3.6. THEOREM. Let $X$ be a locally compact $\sigma$-compact metric space with no isolated points. Then each set $M \subset X$ of the second category in every neighborhood of $X$ contains $c$ mutually disjoint sets, each of the second category in every neighborhood of $X$. 
Proof. Let $B_{1}, B_{2}, \ldots, B_{n}, \ldots$ be the countable basis of open sets with rational radii. We proceed by induction.

By Lemma 3.5 given $U$ an open set in $X$ there exists a set $Q_{1} \subset M \cap \bar{U}$ such that $M-Q_{1}$ is of the second category in every neighborhood in $X$. Let $\bar{U}=\bar{B}_{1}$.

Suppose now that sets $Q_{1}, \ldots, Q_{n-1}$ have been defined so that $M-\left(Q_{1}\right.$ $\left.\cup \ldots \cup Q_{n-1}\right)$ is of the second category in each neighborhood in $X$. By 3.5 there exists a set $Q_{n}$ of the second category contained in $\left\{M-\left(Q_{1} \cup \ldots \cup\right.\right.$ $\left.\left.Q_{n-1}\right)\right\} \cap \bar{B}_{n}$ such that

$$
\left\{M-\left(Q_{1} \cup \ldots \cup Q_{n-1}\right)\right\}-Q_{n}=M-\left\{Q_{1} \cup \ldots \cup Q_{n}\right\}
$$

is of the second category in every neighborhood. The sets $\left\{Q_{n}\right\}$ thus defined obviously satisfy

(i) $Q_{n} \subset \bar{B}_{n} \cap M \quad n=1,2, \ldots$ and are mutually disjoint.

By the theorem of S. Ulam, each $Q_{n}$ contains c mutually disjoint sets each of the second category. Let $\Omega$ be the first uncountable ordinal. We denote the subsets of $Q_{n}$, whose existence we've shown, by $\left\{Q_{n}^{\xi}: \xi<\Omega\right\}$. Then

(ii) $Q_{n}^{\xi} \subset Q_{n} \subset \bar{B}_{n} \cap M$, for $\xi<\Omega \quad n=1,2, \ldots$

(iii) $Q_{n}^{\xi} \cap Q_{n}^{\eta}=\varnothing$ for $\xi<\eta<\Omega n=1,2,3, \ldots$

We define

(iv) $M^{\xi}=\cup_{n=1}^{\infty} Q_{n}^{\xi}$ for each $\xi<\Omega$.

The set $Q_{n}^{\xi} \subset \bar{B}_{n}$ is of the second category. Thus $M^{\xi}$ is of the second category in every neighborhood in $X$ since $B_{n}$ is a basis for the topology of $X$. Obviously $M^{\xi} \subset M$ for $\xi<\Omega$.

Since each of the sets $Q_{1}, \ldots, Q_{n}, \ldots$ are mutually disjoint, (ii) implies that $Q_{m}^{\xi} \cap Q_{n}^{\eta}=\phi$ for $m \neq n, \xi<\Omega, \eta<\Omega$. Thus $M^{\xi} \cap M^{\eta}=\phi$ for $\xi<\eta$ $<\Omega$, proving the theorem.

3.7. COROllary. Each nondiscrete o-compact locally compact metric group may be written as a disjoint union of $\mathrm{c}$ mutually disjoint sets each of the second category in every open set.

4. Haar measure and uniform continuity in topological groups. In this section we show that the continuity of the Haar measure on nondiscrete locally compact groups takes the part of the metric in a metric space. This fact should be evident from the work of the first two sections. Below we give a new proof of the theorem of J. M. Kister [6]. The method of proof has been used by others in metric spaces, and was suggested to the author in the 
case of metric spaces by Professor C. Chou. The proof for metric spaces rests on the fact that it is possible to choose a descending sequence of open balls whose intersection contains no open sets (actually only a point).

4.1. THEOREM. Let $G$ be a locally compact nondiscrete noncompact topological group. Then there exists a bounded real valued function $f$ that is continuous on $G$ but not left uniformly continuous on $G$.

Proof. Let $U$ be a neighborhood of the identity such that $\bar{U}$ is compact. By induction choose a sequence $x_{1}, x_{2}, \ldots, x_{n}, \ldots$ such that $x_{n} \notin$ $\cup_{i=1}^{n-1} x_{i} U$. Let $B$ be a symmetric neighborhood of $e$ satisfying $V \subset U$. Then the sets $\left\{x_{i} V: i \geq 1\right\}$ are pairwise disjoint. Since $G$ is not discrete there is a sequence $V_{1}, V_{2}, \ldots, V_{n}, \ldots$ of nested neighborhoods of $e$ satisfying $\lambda\left(V_{i}\right) \rightarrow 0$. We may choose $V_{1}=V$ without loss of generality. We define now functions $f_{i}, i \geq 1$, as follows:

$$
f_{i}\left(x_{i}\right)=1, \quad f_{i}\left(\mathbb{C} x_{i} V_{i}\right)=\{0\}, \quad 0 \leq f_{i} \leq 1 .
$$

Such functions always exist because $G$ is completely regular. Let $f=$ $\sum_{i=1}^{\infty} f_{i}$. Then $f$ is continuous. However $f$ is not left uniformly continuous. To see this, suppose that given $\varepsilon>0$ we have that there exists $W$ a neighborhood of $e$ such that $x^{-1} y \in W$ implies that $|f(x)-f(y)|<\varepsilon$. Without loss of generality we may suppose $W \subset V$. Observe that if $\varepsilon<1$, and if $x \in$ $x_{n} W$ then $\left|f(x)-f\left(x_{n}\right)\right|<\varepsilon$. This means that $x \in x_{n} V_{n}$ so that $x_{n} W \subset$ $x_{n} V_{n}$ and hence $W \subset V_{n}$. But then $W \subset \cap V_{n}$, contradicting the fact that $\lambda\left(V_{i}\right) \rightarrow 0$.

REMARK. It was pointed out by the referee that the above proof is similar to though simpler than the proof of Theorem 4.10, page 68, in R. B. Burkel [1]. This theorem is as follows: "If $G$ is a locally compact topological group then $W(G)=C(G)$ if and only if $G$ is compact." Here $W(G)$ is the set of weakly almost periodic functions on $G$, and $C(G)$ is the set of continuous functions on $G$. Our construction yields a simpler proof of the part of Burkel's theorem that deals with the nondiscrete case. We also note that the construction in Burkel also proves our Theorem 4.1.

Finally we observe that the method of proof used in 4.1 has actually been anticipated by Comfort and Ross [2]. Specifically their Theorems 1.2 and 2.2 employ a similar construction of a continuous function. 
4.2. EXAMPle. The function $f=\Sigma_{i} f_{i}$, where

$$
f_{i}\left(x_{i}\right)=i, \quad f_{i}\left(\complement x_{i} V_{i}\right)=\{0\}, \quad 0 \leq f_{i} \leq i,
$$

is an example of a function that is continuous, unbounded, and not uniformly continuous, if $\left\{V_{\mathrm{i}}\right\}, V$ are as above.

4.3. COROLlary (Kister). The following are equivalent for a nondiscrete locally compact topological group $G$ :

(a) G is compact

(b) Every continuous real valued function on $G$ is left uniformly continuous.

The above theorem is concerned only with existence of a continuous function that is not left uniformly continuous. A natural question to ask at this point is whether there is a left uniformly continuous real valued function that is not right uniformly continuous. It is clear that if the right and left structure are equivalent then the answer is obviously negative. However, if the two structures are not equivalent this is a meaningful question. We show next that if the group is not unimodular then such functions always exist. The construction is similar to the above one but the technical details are slightly more complicated.

Let $R^{*}$ denote the multiplicative group of positive real numbers.

Definition. Let $\lambda$ be the Haar measure on $G$. The modular function $\triangle: G \rightarrow R^{*}$ is defined by

$$
\Delta(x)=\frac{\int_{G} f\left(t x^{-1}\right) \lambda(d t)}{\int_{G} f(t) \lambda(d t)}, \quad f \in C_{00}^{+}(G) .
$$

$G$ is unimodular iff $\triangle \equiv 1$.

Properties.

(i) $\triangle$ is a continuous homomorphism

(ii) $\triangle(x)=\lambda(E x) / \lambda(E)$ where $E \subset G$ is such that $\lambda(E)>0$. 
4.4. THEOREM. Each locally compact, nondiscrete, nonunimodular group admits a function that is left uniformly continuous but not right uniformly continuous.

Proof. Since $G$ is not unimodular we have that $\Delta$ and $1 / \triangle$ are unbounded on $G$. Let $U$ be a compact symmetric neighborhood of $e$. Inductively we can choose a sequence $\left\{x_{n}\right\} \subset G$ such that

$$
\text { (a) } x_{n} \notin U_{n-1}=\bigcup_{i=1}^{n-1} U x_{i} U
$$

and

$$
\text { (b) } \triangle\left(x_{n}\right)>n \text {. }
$$

To see this observe that $U_{n-1}$ is compact so $G \nsubseteq U_{n-1}$. Also $\triangle\left(U_{n-1}\right)$ is a compact subset of $(0, \infty)$, so there is a point $x_{n} \in G$ such that $\Delta\left(x_{n}\right)>n$ and $\triangle\left(x_{n}\right) \notin \triangle\left(U_{n-1}\right)$.

Now let $V \subset U$ be a symmetric neighborhood of $e$ satisfying $V^{2} \subset U$. Then it is easily seen that each of the following hold

(i) $x_{i} V \cap x_{j} V=\varnothing \quad i \neq j$

(ii) $V x_{i} \cap V x_{j}=\varnothing \quad i \neq j$

(iii) $V x_{i} \cap x_{j} V=\varnothing \quad i \neq j$.

Let $f_{1}(x)$ be any continuous function such that

$$
f_{1}\left(x_{1}\right)=1, \quad f_{1}\left(\mathrm{C}\left[x_{1} V\right]\right)=\{0\}, \quad 0 \leq f_{1} \leq 1 .
$$

Define, for each $i>1$,

$$
f_{i}(x)=f_{1}\left(x_{1} x_{i}^{-1} x\right)
$$

so that $f_{i}\left(x_{i}\right)=f_{1}\left(x_{1}\right)=1$ and

$$
f_{i}\left(\boldsymbol{C} x_{i} V\right)=\{0\} \text {. }
$$

Observe that the function $f=\sum_{i=1}^{\infty} f_{i}$ is continuous and even left uniformly continuous on $G$. However $f$ is not right uniformly continuous. For if $f$ were right uniformly continuous then given $1>\varepsilon>0$ there is $W$ a neighborhood of $e$ such that $x y^{-1} \in W$ implies that

$$
|f(x)-f(y)|<\varepsilon
$$


Without loss of generality we may suppose that $W \subset V$. Then if $x \in W x_{n}$ we have $x \notin V x_{m}, x \notin x_{m} V(m \neq n)$ and $\left|f(x)-f\left(x_{n}\right)\right|<\varepsilon$. Defining $V_{n}$ $=x_{n} V x_{n}^{-1}$ we see that $x \in x_{n} V=V_{n} x_{n}$ and hence $W \subset V_{n}$. Since $n$ is arbitrary we have $W \subset \cap_{n \geq 1} V_{n}$. Observe now that

$$
0<\lambda(V)=\lambda\left(x_{n} V\right)=\lambda\left(V_{n} x_{n}\right)=\lambda\left(V_{n}\right) \Delta\left(x_{n}\right)
$$

Since $\Delta\left(x_{n}\right) \rightarrow \infty$, we must have $\lambda\left(V_{n}\right) \rightarrow 0$. But then $\lambda(W)=0$, a contradiction. Therefore $f$ is not right uniformly continuous.

REMARK. This construction shows that in Theorem 4.1. the function $f$ might possibly be right uniformly continuous if $G$ is not unimodular.

4.5. THEOREM. If $G$ is a nondiscrete locally compact metric group with inequivalent uniform structures then $G$ admits a function $f$ that is left uniformly continuous but not right uniformly continuous.

Proof. It is well-known (see [2], 8.18) that $G$ has equivalent uniform structures iff given $x_{n} \rightarrow e$ and $\left\{y_{n}: n \geq 1\right\}$ any sequence in $G$ then $y_{n}^{-1} x_{n} y_{n}$ $\rightarrow e$. Thus if $G$ has inequivalent uniform structures, there is a neighborhood $U$ of $e$, a sequence $\left\{y_{n}\right\} \subset G$, and a sequence $x_{n} \rightarrow e$, such that $y_{n}^{-1} x_{n} y_{n} \notin$ $U$ for each $n$.

We observe now that $\left\{y_{n}: n \geq 1\right\}$ is not contained in any compact subset $F$ of $G$. This follows from the well known theorem (see $[4], 4.9)$ that given any neighborhood $U$ of $e$ and compact set $F$ there is a neighborhood $V$ of $e$ such that $x^{-1} V x \subset U$, for all $x \in F$. (The condition $y_{n}^{-1} x_{n} y_{n} \in U$ for each $n$, implies that there is.no neighborhood $V$ of $e$ such that $y_{n}^{-1} V y_{n} \subset U$, for all $n$ ).

Without loss of generality we may assume that $\bar{U}$ is compact. Passing to subsequences if necessary we may assume that $y_{n}$ satisfies

$$
y_{n} \notin \bigcup_{i=1}^{n-1} \bar{U} y_{i} \bar{U}, \quad n=2,3,4, \ldots
$$

Let $V \subset U$ satisfy $V^{2} \subset U$. Then as in 4.4 we have

(i) $\left\{V y_{i}: i \geq 1\right\}$ are pairwise disjoint

(ii) $\left\{y_{i} V: 1 \geq 1\right\}$ are pairwise disjoint

(iii) $y_{i} V \cap V y_{j}=\phi$ if $i \neq j$

Define $f$ as in 4.4. As before let $V_{n}$ be defined by $y_{n} V=V_{n} y_{n}$ and suppose $f$ is right uniformly continuous. Then given $\varepsilon>0$ there is a neighborhood $W$ such that $x y^{-1} \in W$ implies that $|f(x)-f(y)|<\varepsilon$. Since we may suppose 
$W \subset V$ we see as in 4.4 that $W \subset V_{n}$, for each $n$. Thus $W \subset \cap V_{n}$. Clearly this implies

$$
y_{n}^{-1} W y_{n} \subset y_{n}^{-1} V_{n} y_{n}=V \subset U
$$

for all $n$. But then this contradicts the statement that there is no neighborhood $V$ of $e$ such that $y_{n}^{-1} V y_{n} \subset U$ for all $n$. Therefore $f$ is not right uniformly continuous.

REMARK. It appears from this last theorem that the classes of left and right uniformly continuous real valued functions on a locally compact topological group with inequivalent uniform structures cannot coincide. However the author was not able to use this construction to prove this. What is clear is that the construction here may be used provided either

(i) $\lambda\left(\cap V_{n}\right) \rightarrow 0$

or

(ii) $\cap V_{n}$ contains no open sets for some sequence of points $\left\{x_{n}\right\} \subset G$ such that

$$
V_{n}=x_{n} V x_{n}^{-1}
$$

\section{REFERENCES}

1. R. B. Burkel, Weakly almost periodic functions on semigroups, 1970.

2. W. W. Comfort and K. A. Ross, Pseudocompactness and uniform continuity in topological groups, Pacific J. Math., 16 (1966), 483-496.

3. P. R. Halmos, Measure Theory, D. Van Nostrand, 1950.

4. E. Hewitt and K. A. Ross, Abstract harmonic analysis, Vol. I, Heidelberg, Springer-Verlag, 1963.

5. S. Kakutani and K. Kodaira, Uber das Haarshe Mass in der lokal bikompaktan Gruppe, Proc. Imp. Acad. Tokyo, 22 (1944), 444-450.

6. J. M. Kister, Uniform continuity and compactness in topological groups, Proc. Amer. Math. Soc., 13 (1962), 37-40.

7. M. Rajagopalan, Fourier transform in locally compact groups, Acta Sci. Math. (Szeged) 25 (1964), 86-89.

8. W. Sierpinski, Sul les ensembles partout de duxieme categorie, Fund. Math., 22 (1934), 1-3.

9. S. Ulam, Zur Masstheorie in der allgemeinen Mengenlehre, Fund. Math. 16 (1930), 140-150.

10. _ Uber gewisse zerlegungen von mengen, Fund. Math., 20 (1933), 221-223.

Received April 8, 1971 and in revised form August 21, 1974. 



\section{PACIFIC JOURNAL OF MATHEMATICS}

\section{EDITORS}

RICHARD ARENS (Managing Editor)

University of California

Los Angeles, California, 90024

\section{R. A. BEAUMONT}

University of Washington

Seattle, Washington 98105

\section{J. DugunDJI}

Department of Mathematics University of Southern California Los Angeles, California 90007

D. Gillbarg AND J. MilgRAM

Stanford University Stanford, California 94305

\section{ASSOCIATE EDITORS}

E. F. BECKENBACH

B. H. NEUMANN

SUPPORTING

UNIVERSITY OF BRITISH COLUMBIA

UNIVERSITY OF CALIFORNIA

MONTANA STATE UNIVERSITY

UNIVERSITY OF NEVADA

NEW MEXICO STATE UNIVERSITY

OREGON STATE UNIVERSITY

UNIVERSITY OF OREGON OSAKA UNIVERSITY
F. WOLF

K. YosHIDA

INSTITUTIONS

UNIVERSITY OF SOUTHERN CALIFORNIA

STANFORD UNIVERSITY

UNIVERSITY OF TOKYO

UNIVERSITY OF UTAH

WASHINGTON STATE UNIVERSITY

UNIVERSITY OF WASHINGTON

AMERICAN MATHEMATICAL SOCIETY

NAVAL WEAPONS CENTER

The Supporting Institutions listed above contribute to the cost of publication of this Journal, but they are not owners or publishers and have no responsibility for its content or policies.

Mathematical papers intended for publication in the Pacific Journal of Mathematics should be in typed form or offset-reproduced, (not dittoed), double spaced with large margins. Underline Greek letters in red, German in green, and script in blue. The first paragraph or two must be capable of being used separately as a synopsis of the entire paper. Items of the bibliography should not be cited there unless absolutely necessary, in which case they must be identified by author and Journal, rather than by item number. Manuscripts, in duplicate if possible, may be sent to any one of the five editors. Please classify according to the scheme of Math. Rev. Index to Vol. ${ }^{39}$. All other communications to the editors should be addressed to the managing editor, or Elaine Barth, University of California, Los Angeles, California, 90024.

100 reprints are provided free for each article, only if page charges have been substantially paid. Additional copies may be obtained at cost in multiples of 50 .

The Pacific Journal of Mathematics is issued monthly as of January 1966. Regular subscription rate: $\$ 72.00$ a year (6 Vols., 12 issues). Special rate: $\$ 36.00$ a year to individual members of supporting institutions.

Subscriptions, orders for back numbers, and changes of address should be sent to Pacific Journal of Mathematics, 103 Highland Boulevard, Berkeley, California 90708.

\section{PUBLISHED BY PACIFIC JOURNAL OF MATHEMATICS, A NON-PROFIT CORPORATION}

Copyright (C) 1974 by Pacific Journal of Mathematics

Manufactured and first issued in the U.S.A. 


\section{Pacific Journal of Mathematics}

\section{Vol. 54, No. $2 \quad$ June, 1974}

John Edward Coury, Walsh series with coefficients tending monotonically to

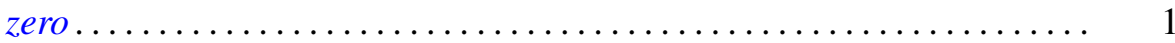

Patrick Michael Fitzpatrick and Walter Volodymyr Petryshyn, Fixed point theorems for multivalued noncompact acyclic mappings ............

Irving Leonard Glicksberg, More on Phragmén-Lindelöf for function

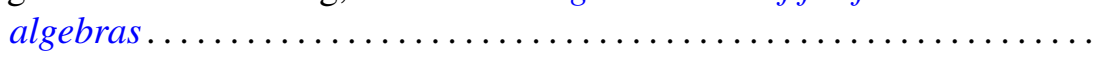

Adilson Goncalves, Structural constants. II .................. 39

Richard P. Gosselin, Closure theorems for affine transformation groups .... 53

Ralph Peter Grimaldi, Baer and UT-modules over domains ........... 59

Edward Grossman, On the prime ideal divisors of $\left(a^{n}-b^{n}\right) \ldots \ldots \ldots \ldots . \ldots 73$

A. Hedayat and Ester Seiden, On the theory and application of sum composition of Latin squares and orthogonal Latin squares.......... .

Gerald L. Itzkowitz, Continuous measures, Baire category, and uniform continuity in topological groups ......................... 115

Francis Masat, Right simple congruences on a semigroup ............ 127

Robert Harvey Oehmke, Right congruences and semisimplicity for Rees matrix semigroups..................................

Qazi Ibadur Rahman and Jan Stankiewicz, Differential inequalities and local valency . . . . . . . . . . . . . . . . . . . . . . . . . . . . . . . . . . . . . 165

William John Reed, Random points in a simplex ................ 183

Mohan S. Shrikhande, Strongly regular graphs and group divisible

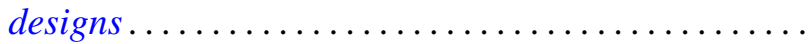

Zahava Shmuely, The structure of Galois connections ... . .

Robert C. Shock, Dual generalizations of the Artinian and Noetherian

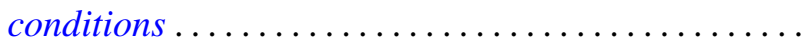

Arne Stray, Approximation and interpolation for some spaces of analytic functions in the unit disc ........................

Eldon Jon Vought, Monotone decompositions into trees of Hausdorff continua irreducible about a finite subset ............

James Wirth, The mapping cylinder axiom for WCHP fibrations ... 263

Gordon S. Woodward, Invariant means and ergodic sets in Fourier analysis... 